\title{
Assessing Bladder Cancer Risk in Type 2 Diabetes Clinical Trials: the Dapagliflozin Drug Development Program as a 'Case Study'
}

\author{
Agata Ptaszynska · Samuel M. Cohen · Edward M. Messing • \\ Timothy P. Reilly · Eva Johnsson · Kristina Johnsson
}

To view enhanced content go to www.diabetestherapy-open.com

Received: June 8, 2015 / Published online: September 1, 2015

(C) The Author(s) 2015. This article is published with open access at Springerlink.com

\section{ABSTRACT}

Introduction: Dapagliflozin, a sodium-glucose co-transporter 2 inhibitor, decreases plasma glucose levels by suppressing renal glucose reabsorption and increasing urinary glucose excretion. Previously published pre-clinical data suggest that dapagliflozin lacks carcinogenic potential. This article reviews data on bladder cancer with dapagliflozin to illustrate the challenges in assessing bladder cancer in drug development programs in patients with type 2 diabetes mellitus (T2DM).

Electronic supplementary material The online version of this article (doi:10.1007/s13300-015-0128-9) contains supplementary material, which is available to authorized users.

A. Ptaszynska $(\varangle) \cdot$ T. P. Reilly

Bristol-Myers Squibb, Princeton, NJ, USA

e-mail: agata.ptaszynska@bms.com

S. M. Cohen

University of Nebraska Medical Center, Omaha, NE, USA

E. M. Messing

Department of Urology, University of Rochester

School of Medicine and Dentistry, Rochester,

NY, USA

E. Johnsson · K. Johnsson

AstraZeneca, Mölndal, Sweden
Methods: Clinical cases of bladder cancer were analyzed in a pooled population of $>9000$ patients in 21 phase $2 \mathrm{~b} / 3$ dapagliflozin clinical trials of up to 208 weeks' duration.

Results: In the 21-study pool, demographic and baseline characteristics were generally consistent between dapagliflozin and comparator groups. The overall incidence of malignancies was also balanced between the treatment groups, with an incidence rate ratio (IRR) of 1.035 [95\% confidence interval (CI): 0.724, 1.481]. Nine of 5936 dapagliflozin-treated patients and 1 of 3403 comparator-treated patients reported bladder cancer, with an IRR of 5.168 (95\% CI: 0.677, 233.55). All of these patients had clinical attributes typical of bladder cancer in the general population ( $\geq 60$-year-old males; 8 of the 10 patients were current/former smokers). All cases of bladder cancer were reported within 2 years of starting study treatment. There was an absence of detailed workup of hematuria prior to randomization, and no hematuria workup data were collected proactively in the dapagliflozin trials, which is typical of clinical practice. Failure to exclude bladder cancer prior to randomization increases the chance of 
recruiting patients with pre-existing bladder cancer in clinical trials and may delay the final diagnosis. Of the nine dapagliflozin-treated patients with bladder cancer, eight had microscopic hematuria prior to start of treatment or within 6 months of initiating study treatment.

Conclusion: The assessment of bladder cancer data illustrates the challenges of characterizing cancer risk in T2DM drug development programs. The totality of evidence to date does not suggest a causal relationship between dapagliflozin and bladder cancer.

Funding: AstraZeneca.

Keywords: Bladder cancer; Dapagliflozin; Hematuria; SGLT2 inhibitor; Type 2 diabetes mellitus

\section{INTRODUCTION}

Epidemiologic data suggest that people with diabetes are at a significantly higher risk of many forms of cancer [1]. However, it is not clear if diabetes itself is an independent risk factor for cancer, or if the association can be explained by common risk factors such as obesity and a sedentary lifestyle shared between diabetes and several forms of cancer. Specifically, data show that type 2 diabetes mellitus (T2DM) may be associated with an increased risk of bladder cancer, with a meta-analysis showing 35\% increased risk in patients with diabetes versus a healthy population, [2, 3], although this positive association may be limited to patients with longstanding diabetes and/or to insulin users [4, 5]. One study reported increased odds of bladder cancer in patients with T2DM of $\geq 16$ years' standing versus non-diabetics [odds ratio 3.6; 95\% confidence interval (CI): 1.1, 11.2] [5].
Against this background, the influence of glucose-lowering drugs on cancer risk is of great interest, and several therapies have been under scrutiny because of signals for potential increased cancer risk. Due to the relative infrequency of cancer events, detecting a cancer signal in pre-marketing clinical development programs is very difficult. Therefore, assessment should be based on the totality of evidence, including pre-clinical and clinical data, individual case reports, as well as continued monitoring for any potential signals in post-marketing surveillance and longer-term clinical studies.

Data suggest that long-term use of insulin glargine ( $>5$ years) may increase the risk of breast cancer [6]. However, findings from the ORIGIN trial (Outcome Reduction with Initial Glargine Intervention; ClinicalTrials.gov \#NCT00069784) [7], which enrolled 12,537 patients and followed them for a median of 6.2 years, found no increased breast cancer risk with insulin glargine versus standard therapy [8, 9]. Further, a potential link between glucagon-like peptide-1 receptor agonists (GLP-1 RAs) and pancreatic carcinoma and thyroid cancer has also been of concern in recent years. However, no clear conclusions have been drawn regarding a causal relationship with pancreatic disease (pancreatic cancer and pancreatitis) [10-13]. With regard to thyroid cancer, clinical data have not shown any association between GLP-1 RAs and thyroid cancer in patients with T2DM [14].

There have also been some concerns about the potential association between the thiazolidinedione pioglitazone [a peroxiosome proliferator-activated receptor (PPAR) $\gamma$ agonist) and bladder cancer $[15,16]$, which was evaluated in a 10-year epidemiologic study. Although a 5-year interim analysis of this 
study showed a statistically significant increased risk of bladder cancer among patients on pioglitazone for $\geq 2$ years [17], the 10-year final analysis did not show any statistically significant increases [18]. Reflecting this, the updated American Diabetes Association/ European Association for the Study of Diabetes position statement states that earlier concerns about the association of pioglitazone and bladder cancer have been dismissed by evidence provided by recently published clinical studies [19, 20].

More recently, sodium-glucose co-transporter 2 (SGLT2) inhibitors have emerged as a novel class of oral medications for T2DM. Dapagliflozin works by suppressing renal glucose reabsorption and increasing urinary glucose excretion via SGLT2 inhibition, thereby leading to decreases in plasma glucose levels [21]. Dapagliflozin is approved for adults with T2DM in the USA as an adjunct to diet and exercise [22], and in Europe for use as monotherapy or add-on combination therapy [23]. A fixed dose combination of dapagliflozin and metformin is also approved in the USA and Europe as an adjunct to diet and exercise when treatment with both dapagliflozin and metformin is appropriate [24, 25]. Bladder cancer concerns were raised with dapagliflozin due to the observed numerical imbalance between treatment groups in the new drug application submitted to the Food and Drug Administration in 2011; 9 out of 5478 patients on dapagliflozin and 1 out of 3156 patients on control reported bladder cancer in the pooled dapagliflozin studies [26]. Bladder cancer occurrences have not been reported in humans with other SGLT2 inhibitors; while they have been shown to induce tumors (although not urothelial tumors of the kidney pelvis, ureters, or bladder) in rats [27] and male mice [28], the associated mechanisms are not considered relevant to humans.

This article reviews data on bladder cancer with dapagliflozin to illustrate the methodologic challenges in assessing cancer risk in drug development programs in T2DM. The article further highlights complications in the assessment of bladder cancer in the case of SGLT2 inhibitors due to their glucosuric mechanism of action.

\section{PRE-CLINICAL STUDIES OF DAPAGLIFLOZIN}

The potential for SGLT2 inhibition and exposure to dapagliflozin to induce tumors or enhance tumor growth have been examined in pre-clinical studies. This includes studies of cancers in general, as well as bladder cancer in particular.

Pharmacologically, dapagliflozin does not raise any concerns as SGLT2 is not expressed in bladder tissue [29], and the action of dapagliflozin is highly specific to SGLT2, with no off-target effects observed across an in vitro screen of $>330$ receptors, enzymes, ion channels, and transporters [30]. The presence of glucosuria did not enhance background hyperplastic effects, or lead to or enhance bladder tumors in in vivo or in vitro models. Dapagliflozin induced extensive glucosuria in both rodents and dogs that exceeded human urinary glucose concentrations $(166 \mathrm{mM})$ and did not lead to bladder proliferative or tumor development [30, 31]. Furthermore, there was no evidence of hyperplasia or neoplasia in the kidney or bladder of SGLT2 knockout mice with glucosuria of $\sim 2000 \mathrm{mg} / \mathrm{dL}$, compared with its wild-type counterpart with no glucosuria [32].

There was also no evidence of carcinogenic potential for dapagliflozin in toxicology studies. A recent study showed that the exposure of 
CD-1 mice and Sprague-Dawley rats to dapagliflozin for up to 2 years at $\geq 100$-fold human clinical exposure did not increase tumor incidence or bladder proliferative/ preneoplastic lesions [33]. No proliferative or hyperplastic changes were observed in dogs (a species that has been shown to be highly predictive of human bladder carcinogens [34]) over 1 year at $>3000$-fold human clinical exposure of dapagliflozin. In summary, these pre-clinical data suggest that dapagliflozin lacks carcinogenic potential.

\section{CLINICAL DATA FOR DAPAGLIFLOZIN}

\section{Methods}

Cancer cases were analyzed in a pooled population of $>9000$ patients taking part in 21 phase $2 \mathrm{~b} / 3$ dapagliflozin clinical trials ranging from 12 to 208 weeks' duration (five phase $2 \mathrm{~b}$ studies of 12 weeks' duration, and 16 phase 3 studies of 24-208 weeks' duration) (see Table S1 in the Electronic Supplementary Material). Treatment groups included dapagliflozin (2.5, $5,10,20$, and $50 \mathrm{mg}$ ) versus comparator (placebo with or without background medications or active control including benchmark treatments). Overall, only 59 and 56 patients received 20 and $50 \mathrm{mg}$ doses of dapagliflozin, respectively, for 12 weeks, with the remainder receiving dapagliflozin 2.5, 5 and $10 \mathrm{mg}$.

Hematuria was measured at baseline and during clinic visits. The frequency of patient visits during the treatment period was different in individual studies, varying from weekly visit in the beginning to every 4th week in short-term studies, and every 12 th week in long-term studies. Urine dipstick analyses were performed at all visits, and samples with positive dipstick underwent microscopic urinalysis.

Events were identified according to the Standardized MedDRA Query of 'malignant or unspecified tumors'.

The analysis reported in this article is based on previously conducted studies and does not involve any new studies of human or animal subjects performed by any of the authors.

\section{Statistical Analyses}

Incidence rate ratio (IRR) and Kaplan-Meier estimates of events were calculated. Due to the small number of bladder cancer cases, analyses of IRR were estimated using the Cox proportional hazard ratio model stratified by study [35].

\section{Clinical Findings}

The number of patients in the dapagliflozin group ( $n=5936$ ) was much higher than in the comparator group $(n=3403)$. Demographic and baseline characteristics were generally consistent between the two groups (Table 1). Most patients were $<65$ years of age and were overweight or obese; almost $90 \%$ of patients in both groups had a body mass index (BMI) $\geq 25 \mathrm{~kg} / \mathrm{m}^{2}$, while approximately half of all patients had a BMI $\geq 30 \mathrm{~kg} / \mathrm{m}^{2}$. Mean duration of T2DM was 7.0 and 7.6 years in the dapagliflozin and comparator groups, respectively, and mean baseline glycated hemoglobin (HbA1c) values were $8.21 \%$ and $8.14 \%$, respectively. Overall, $43.3 \%$ and $46.3 \%$ of patients in the dapagliflozin and comparator groups, respectively, had a history of smoking. The pooled analysis included patients from an add-on to pioglitazone study $(n=420)$ and 
Table 1 Demographic and baseline characteristics

\section{Dapagliflozin $(n=5936) \quad$ Comparator $(n=3403)$}

Age, years

Mean $\pm S D$

Age categorization, years, $n$ (\%)

$<65$

$\geq 65$ and $<75$

$\geq 75$

Gender, $n(\%)$

Male

Female

Race, $n$ (\%)

White

Black/African American

Asian

Other

BMI, $\mathrm{kg} / \mathrm{m}^{2}, n(\%)$

$$
\geq 25
$$$$
\geq 30
$$

Smoking history, $n$ (\%)

Current

Former

$998(16.8)$

$1572(26.5)$

$6.95 \pm 7.49$

$4.50(0.0,54.4)$

$8.21 \pm 1.03(66 \pm 11.3)$

$167.21 \pm 48.78(9.28 \pm 2.71)$

$130.4 \pm 15.66$

$131.1 \pm 14.88$

$4512(76.0)$

$2424(71.2)$

$859(25.2)$

$120(3.5)$

1964 (57.7)

$1439(42.3)$

$2644(77.7)$

$125(3.7)$

$513(15.1)$

$121(3.6)$

2998 (88.1)

$1949(57.3)$

$562(16.5)$

1014 (29.8)

$7.59 \pm 7.68$

$5.20(0.0,48.0)$

$8.14 \pm 1.02(65 \pm 11.1)$

$165.27 \pm 46.48(9.17 \pm 2.58)$

Seated systolic blood pressure. $\mathrm{mmHg}$

$$
\text { Mean } \pm \text { SD }
$$

Seated diastolic blood pressure, $\mathrm{mmHg}$

Mean \pm SD
$78.8 \pm 8.94$ 
Table 1 continued

\begin{tabular}{lll}
\hline & Dapagliflozin $(\boldsymbol{n}=\mathbf{5 9 3 6})$ & Comparator $(\boldsymbol{n}=\mathbf{3 4 0 3})$ \\
\hline GFR, $\mathrm{mL} / \mathrm{min} / 1.73 \mathrm{~m}^{2}, n(\%)$ & & \\
$<30$ & $9(0.2)$ & $6(0.2)$ \\
$\geq 30$ to $<60$ & $668(11.3)$ & $387(11.4)$ \\
$\geq 60$ to $<90$ & $3113(52.4)$ & $1793(52.7)$ \\
$\geq 90$ & $2146(36.2)$ & $1216(35.7)$ \\
\hline
\end{tabular}

Includes all treated patients from 21 phase $2 \mathrm{~b} / 3$ dapagliflozin clinical trials ranging from 12 to 208 weeks' duration (five phase $2 \mathrm{~b}$ studies of 12 weeks' duration and 16 phase 3 studies with 24-208 weeks' duration)

$B M I$ body mass index, FPG fasting plasma glucose, GFR glomerular filtration rate, HbAlc hemoglobin A1c, SD standard deviation, $T 2 D M$ type 2 diabetes mellitus

from four studies with background insulin and/ or oral antidiabetic drugs $(n=3017)$.

\section{Malignant or Unspecified Tumors}

The overall incidence rate of malignancies was balanced between dapagliflozin and comparator groups; 89/5936 (1.5\%) patients treated with dapagliflozin and 51/3403 (1.5\%) patients treated with comparator had malignant or unspecified tumors. The IRR for overall malignant and unspecified tumors was 1.035 (95\% CI: 0.724, 1.481; $p=0.849$ ). The time-to-first-event analyses of malignant and unspecified tumors (Kaplan-Meier estimates) were also similar between patients treated with dapagliflozin and comparator (Table 2).

When different cancer types were analyzed, the incidence rates were numerically lower in the dapagliflozin group versus comparator for some organ categories (e.g., renal, female reproductive), and greater in the dapagliflozin group versus comparator for other organ categories (e.g., bladder, breast) (Fig. 1). There was no obvious pattern to the distribution of various tumor types, with no clear pathophysiologic link between the tumor types favoring dapagliflozin or comparator. The CIs for the incidence rate for all specific tumor types included unity, indicating that there was no statistically significant difference between treatment groups. The majority of patients with cancer were diagnosed within 1 year of initiating study drug treatment (72\% and $63 \%$ in the dapagliflozin and comparator groups, respectively).

\section{Bladder Cancer}

Nine of 5936 dapagliflozin-treated patients $(0.15 \%)$ and 1 of 3403 comparator-treated patients $(0.03 \%)$ reported bladder cancer events (Table 3). This corresponds to an unstratified incidence rate of 135 events per 100,000 patient-years for dapagliflozin-treated patients and 26 events per 100,000 patient-years for comparator-treated patients, with an IRR of 5.168 (95\% CI: 0.677, 233.55) (Table 4).

All patients reporting bladder cancer were male, most were $\geq 60$ years old, and had clinical attributes typical of bladder cancer in the general population; the pattern of urothelial cell carcinomas detected during the study period was typical of the general population [36], with approximately 50\% being low-grade non-invasive cancer at first diagnosis. Furthermore, 8/10 patients with events of 
Table 2 Cumulative proportion of patients with malignant and unspecified tumors (Kaplan-Meier analysis)

\begin{tabular}{|c|c|c|c|c|c|c|}
\hline \multirow[t]{2}{*}{ Week } & \multicolumn{3}{|c|}{ Dapagliflozin $(n=5936)$} & \multicolumn{3}{|c|}{ Comparator $(n=3403)$} \\
\hline & $\bar{n}$ & $N \#$ & Proportion, \% (95\% CI) & $\bar{n}$ & $N \#$ & Proportion, \% (95\% CI) \\
\hline$<18$ & 24 & 5347 & $0.42(0.25-0.59)$ & 11 & 3070 & $0.34(0.14-0.54)$ \\
\hline$<26$ & 31 & 4846 & $0.57(0.37-0.77)$ & 15 & 2771 & $0.48(0.24-0.73)$ \\
\hline$<52$ & 64 & 3245 & $1.50(1.13-1.88)$ & 31 & 1911 & $1.25(0.80-1.70)$ \\
\hline$<104$ & 85 & 1552 & $2.69(2.06-3.32)$ & 45 & 774 & $2.69(1.81-3.57)$ \\
\hline$<208$ & 89 & 156 & $3.93(2.27-5.59)$ & 50 & 135 & $5.76(2.97-8.54)$ \\
\hline$<216$ & 89 & 3 & 3.93 & 51 & 2 & 6.46 \\
\hline
\end{tabular}

Including data after rescue

$n$ Number of patients with an event of malignant cancer, $N$ number of treated patients, $N \#$ number of patients at risk at the beginning of the period, $C I$ confidence interval

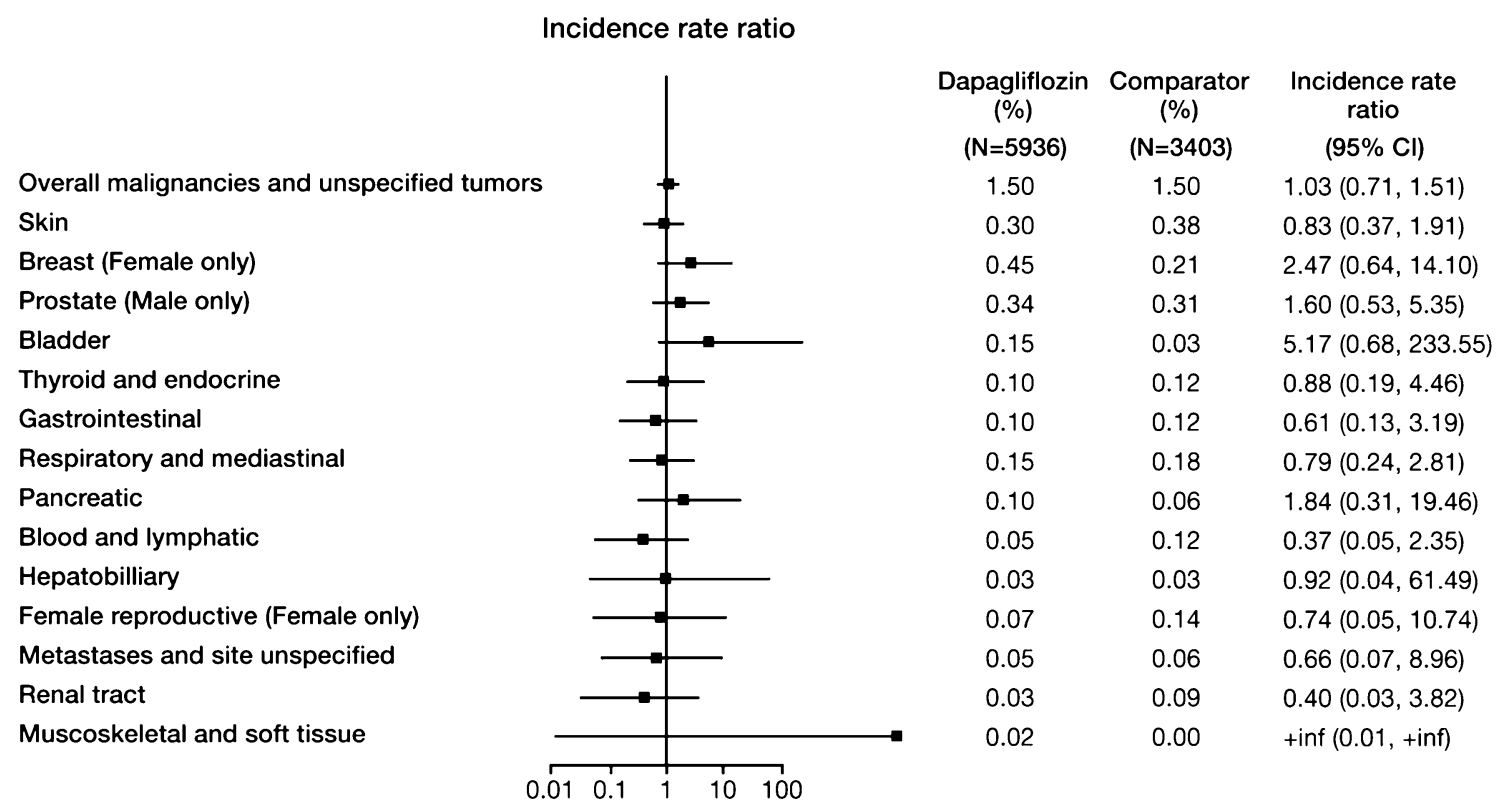

Fig. 1 Incident rate ratio of malignant and unspecified tumors by organ category from the dapagliflozin clinical trials. $N$ is the number of treated patients. Only trials with at least one selected event contributed to the analysis. Confidence intervals are based on an exact method.

bladder cancer were current or former smokers. These findings are consistent with data from previous studies reporting bladder cancer predominance in smokers [37, 38]. It should be noted that history of smoking in patients
Includes serious and non-serious adverse events on or after the first date/time of double-blind treatment and on or prior to the last day of short-term plus long-term treatment +30 days (or up to follow-up visit whichever was first) or +4 days

who reported bladder cancer was higher than in the overall study population of male dapagliflozin- and comparator-treated patients $(58.5 \%$ and $61.1 \%$, respectively, were current or former smokers). 


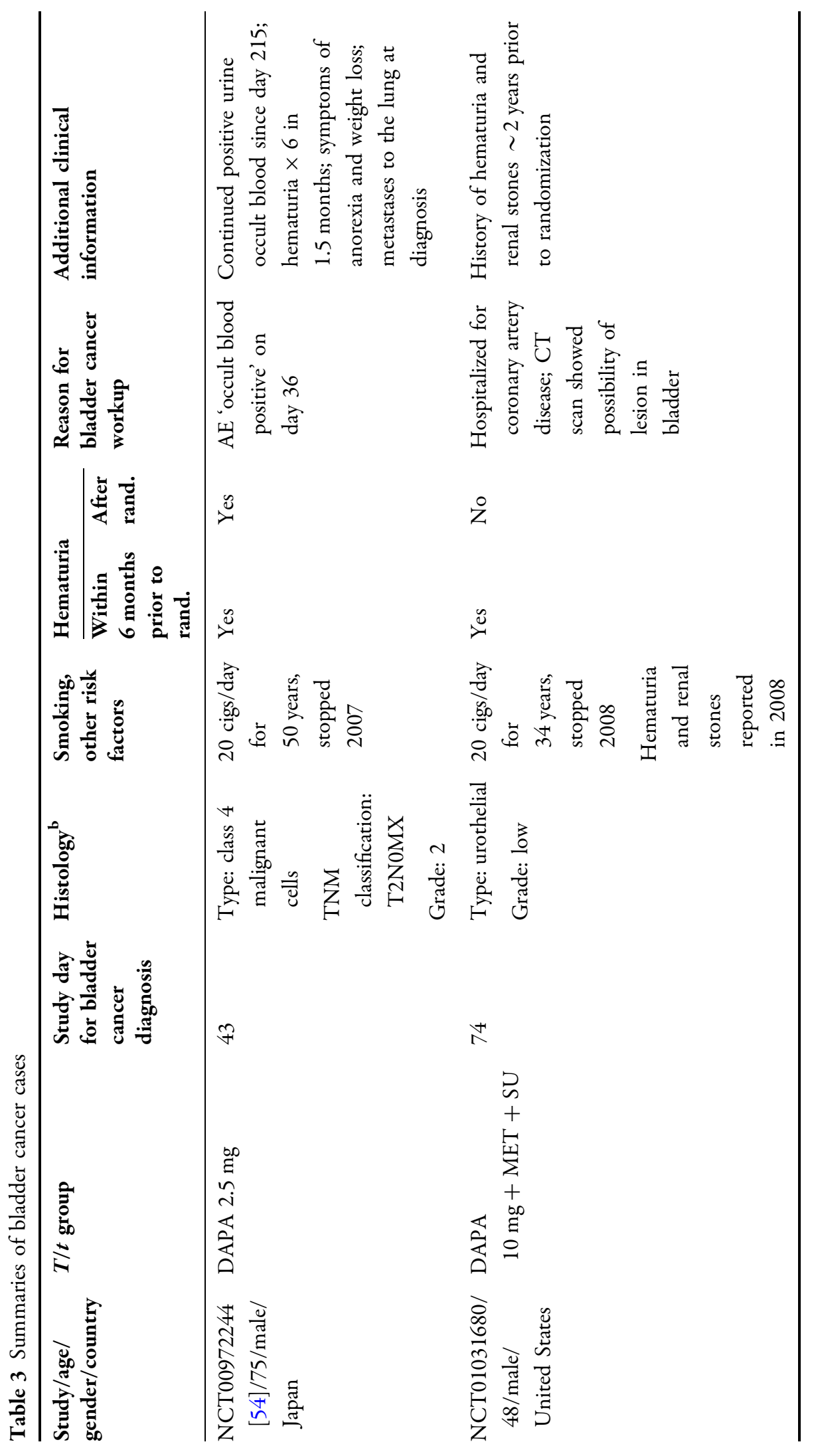




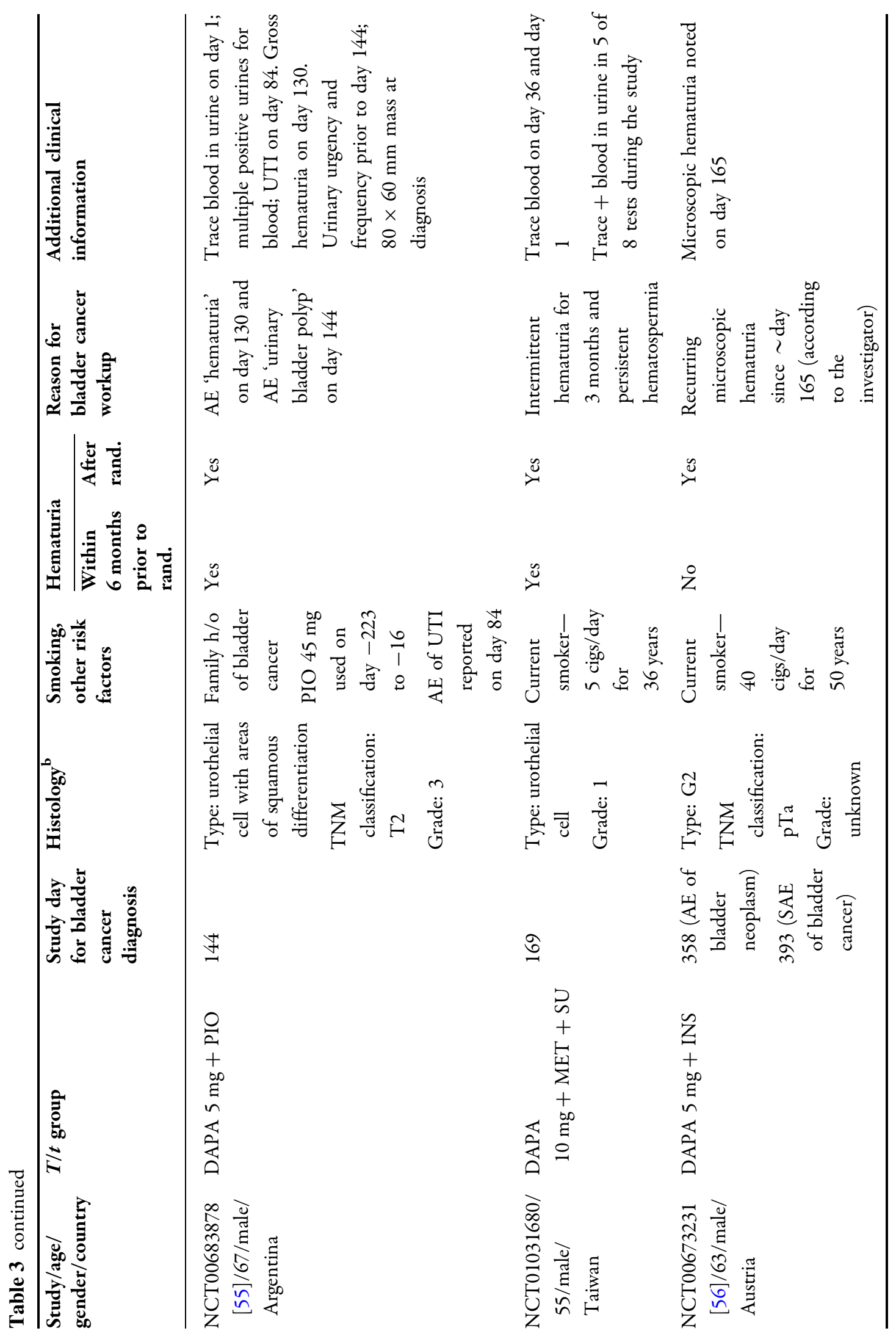




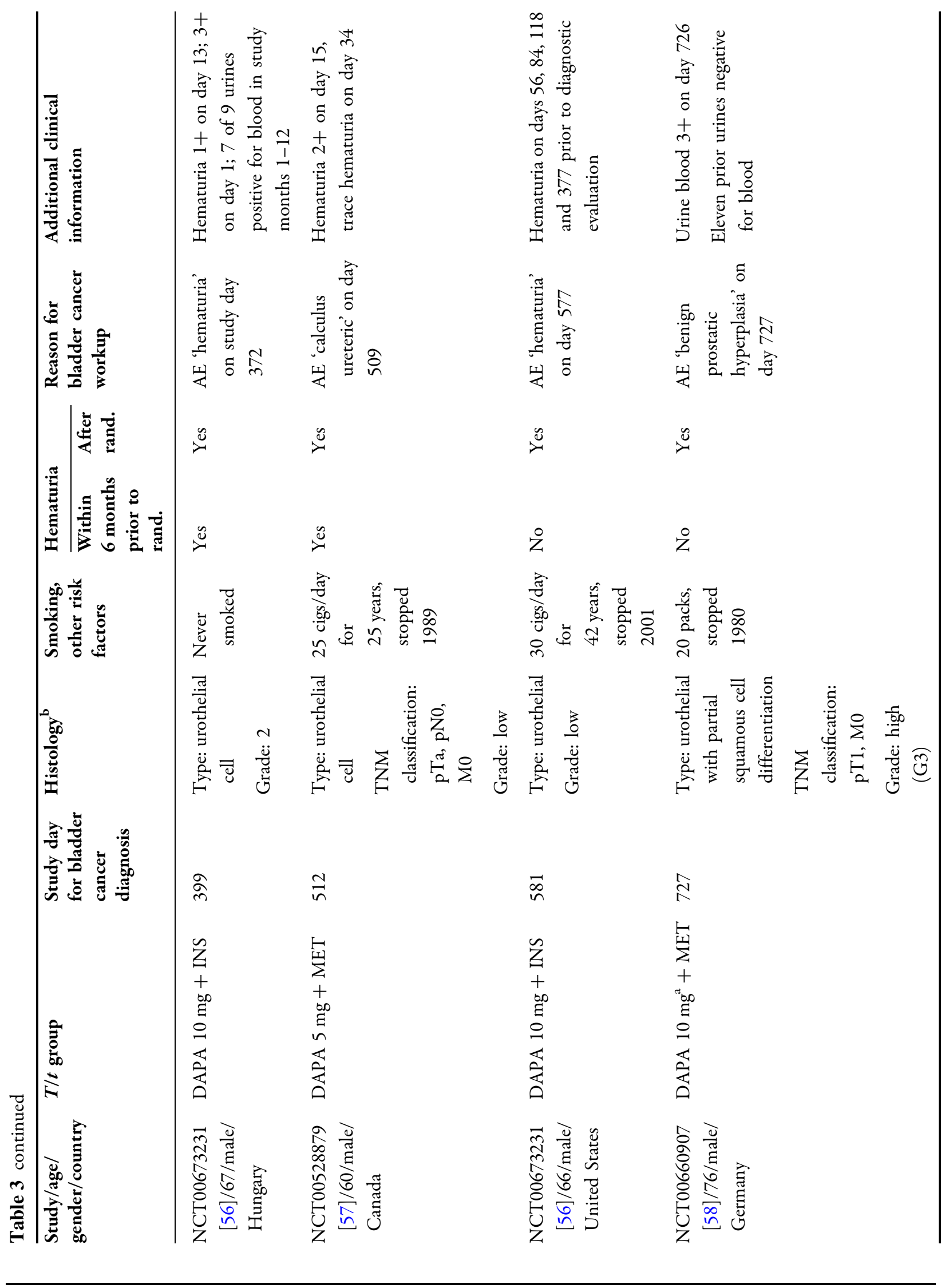




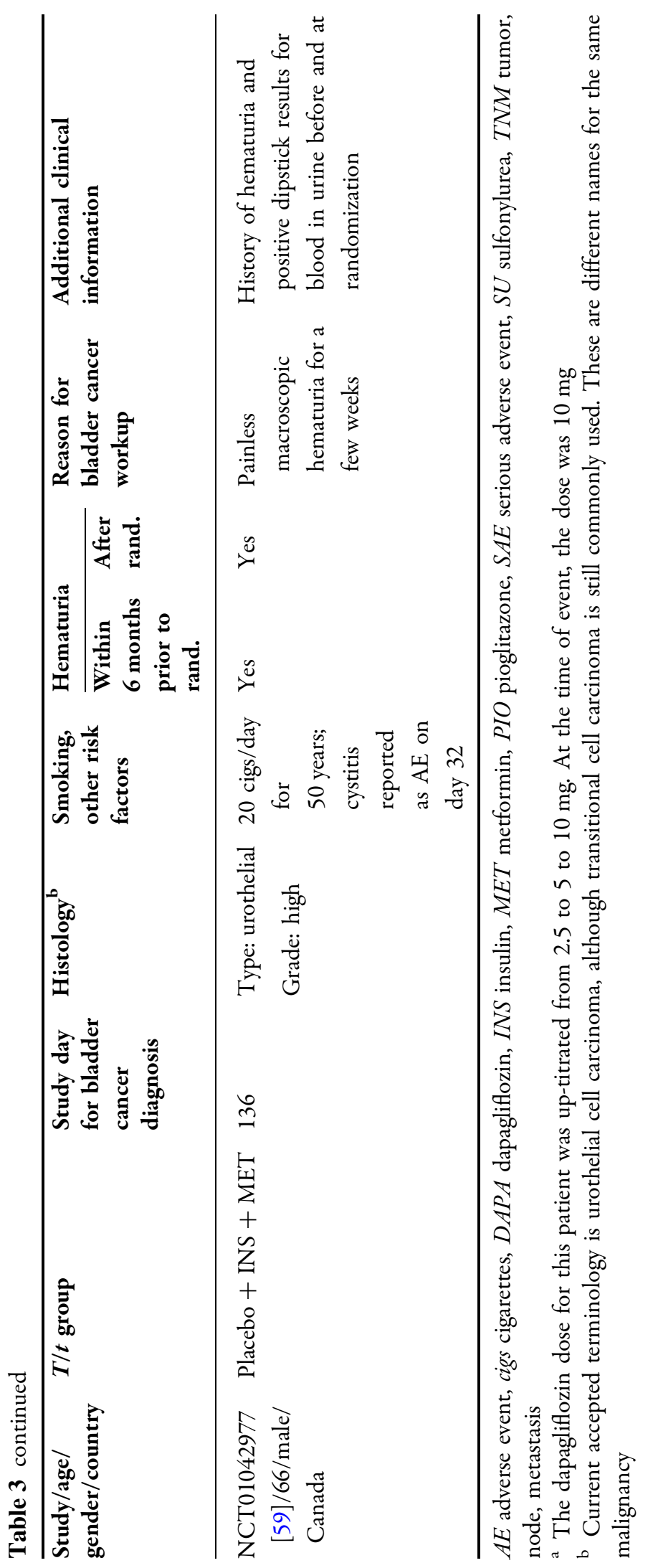


Table 4 Adverse events of bladder cancer, including data after rescue

\begin{tabular}{lll}
\hline & Dapagliflozin $(\boldsymbol{n}=\mathbf{5 9 3 6})$ & Comparator $(\boldsymbol{n}=\mathbf{3 4 0 3})$ \\
\hline Exposure, patient-years & 6650 & 3864 \\
Patients with events, $n(\%)$ & $9(0.15)$ & $1(0.03)$ \\
Incident rate per 100 patient-years & 0.135 & 0.026 \\
Incident rate ratio vs comparator $(95 \% \mathrm{CI})^{\mathrm{a}}$ & $5.168(0.677,233.55)$ & - \\
\hline
\end{tabular}

${ }^{a}$ One additional case of bladder cancer in a patient treated with dapagliflozin $10 \mathrm{mg}$ has been reported since database lock. The subject is a female, $<60$ years of age and a current smoker ( 20 cigarettes/day for 40 years). Bladder cancer was diagnosed within 3.5 months of starting treatment with dapagliflozin suggesting that the cancer was pre-existent. The incidence rate ratio in the dapagliflozin total group versus comparator including this additional event is 6.111 (95\% CI: $0.827,272.02$ )

The hypothesis that symptoms related to increased urinary volume and increased events of urinary tract infections (UTIs) with dapagliflozin treatment led to increased bladder cancer detection bias has not been fully tested. In clinical studies of dapagliflozin, three patients had urinary events prior to bladder cancer diagnosis: one dapagliflozin-treated patient had a UTI on day 84 of the study (bladder cancer was detected on day 144), the second dapagliflozin-treated patient had an adverse event (AE) of benign prostatic hypertrophy on day 727 (the same day as cystoscopy workup for benign prostatic hypertrophy; bladder cancer was detected as an incidental finding), and the third comparator-treated patient had cystitis on day 32 (bladder cancer was detected on day 136).

Eight of the nine bladder cancer patients who received dapagliflozin were treated with background glucose-lowering medications: insulin $(n=3)$, metformin $(n=2)$, metformin and sulfonylurea $(n=2)$, and pioglitazone $(n=1)$. The patient in the comparator group who reported bladder cancer received insulin and metformin background therapy (Table 3).

All ten cases of bladder cancer were reported within 2 years of starting study treatment (range 43-727 days) (Fig. 2). The bladder cancer events were diagnosed in the dapagliflozin-treated patients in $<6$ months $(n=4), \quad 6$ to $<12$ months $(n=1), 12$ to $<18$ months $(n=2)$, and 18 to $<24$ months $(n=2)$ of starting the study drug, which is a relatively short amount of time when considering the latency period for human bladder carcinogenesis [39, 40].

\section{Hematuria and Bladder Cancer}

Hematuria, microscopic or macroscopic, is the most common symptom of bladder cancer. In addition to being a manifestation of bladder cancer, hematuria can also be a sign of carcinoma in situ and may be associated with bladder cancer detected up to 6 years later [41].

Of the nine dapagliflozin-treated patients with bladder cancer, eight had microscopic hematuria prior to start of treatment or within 6 months of initiating study drug treatment (Fig. 3) and one reported hematuria 6 months after initiating study treatment. The patient with bladder cancer in the comparator-treated group had evidence of hematuria prior to start of treatment. It should be noted that in the 


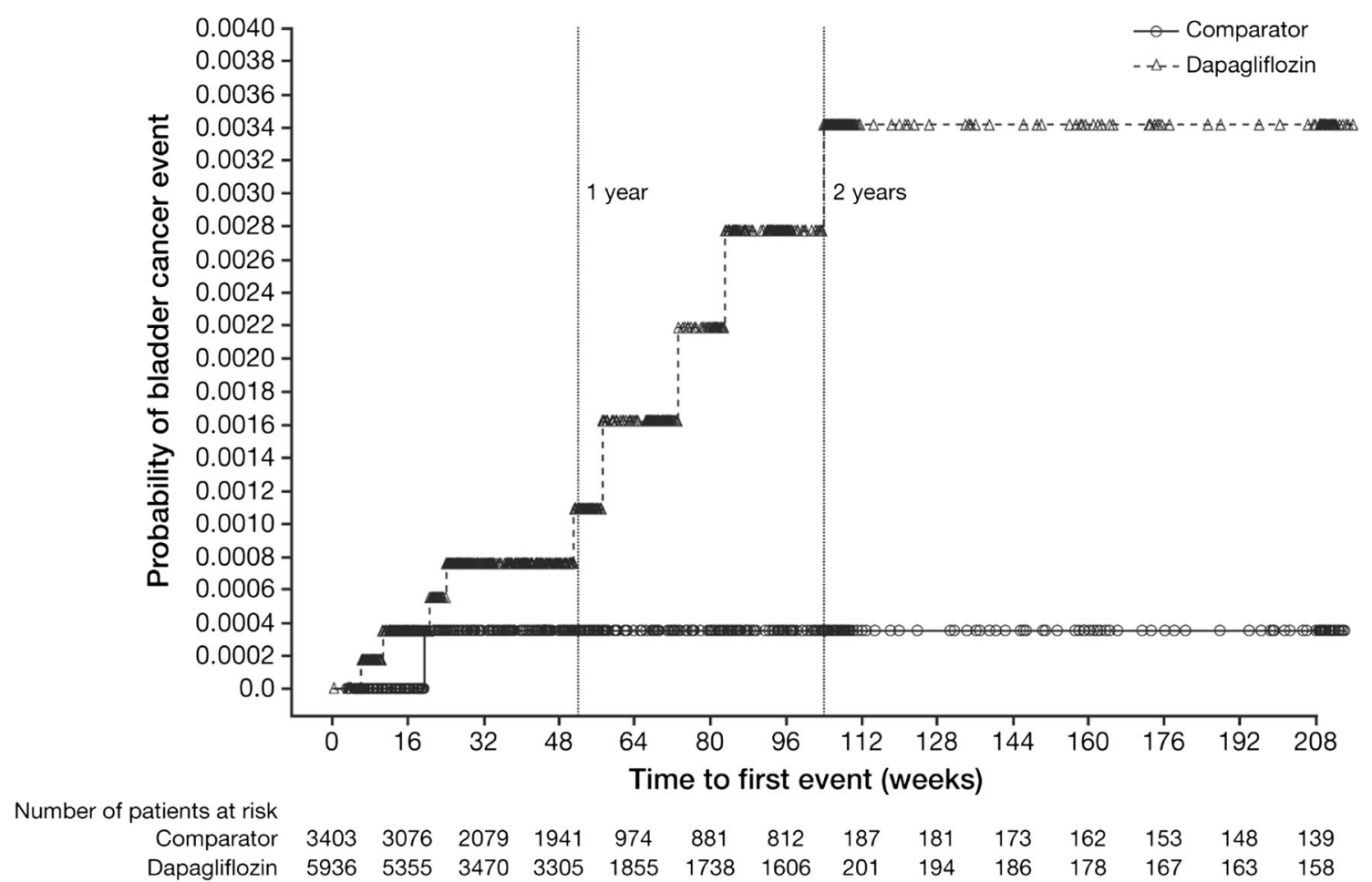

Fig. 2 Kaplan-Meier plot of time to first event of bladder cancer. Includes data after rescue. Week is not scheduled week visit, but actual days from the first dose of double-blind study medication divided by 7 . The number of patients at risk is the number of patients at risk in the beginning of the period

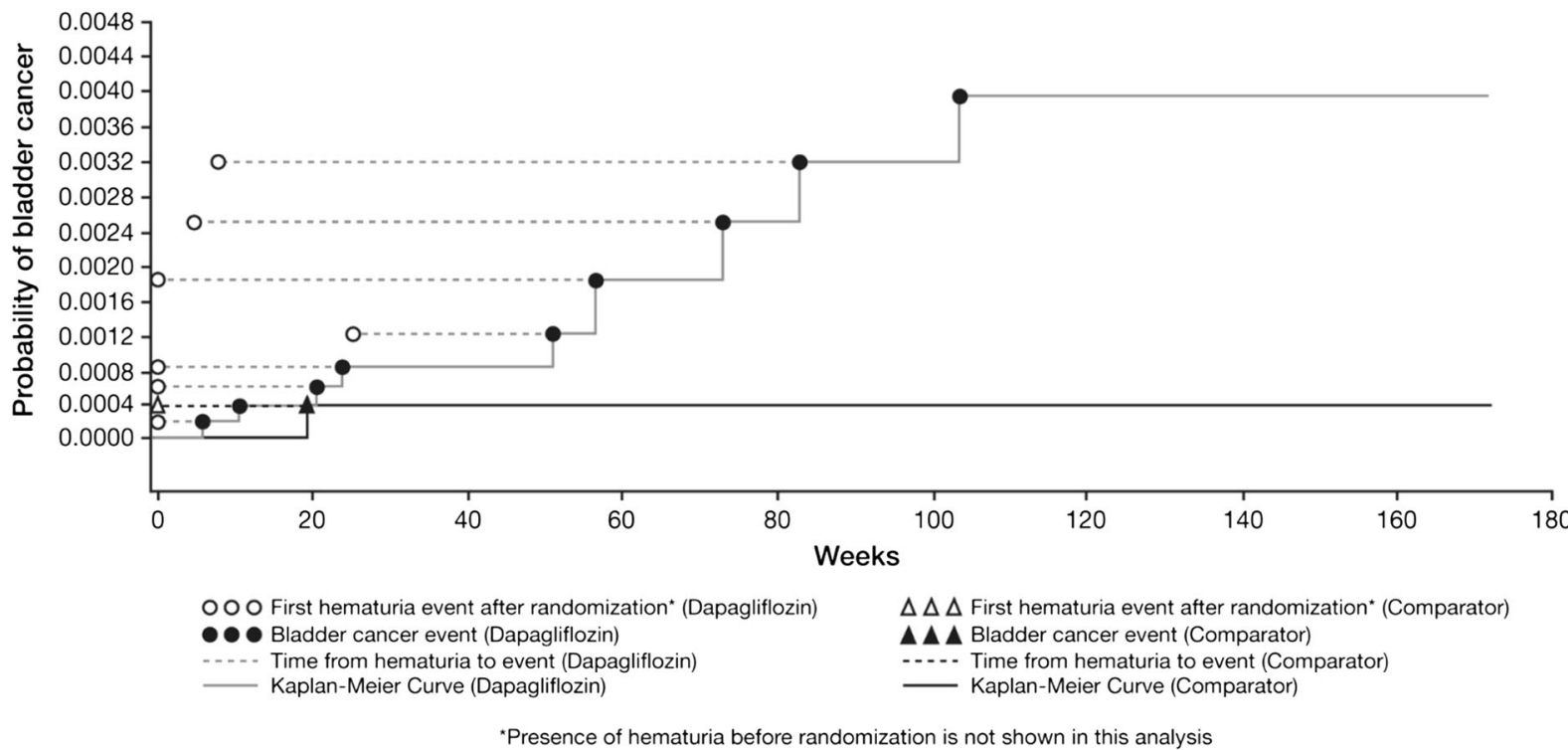

Fig. 3 Relationship between first hematuria event after randomization and time to first bladder cancer event 
overall pool of $>9000$ patients, the prevalence of hematuria at baseline was similar in patients receiving dapagliflozin and comparator $[8.5 \%$ of patients on dapagliflozin $3.7 \%$ of men and $14.2 \%$ of women) versus $8.1 \%$ on comparator (4.2\% of men and $13.4 \%$ of women)].

\section{DISCUSSION}

The assessment of bladder cancer data from the dapagliflozin drug development program illustrates the challenge of characterizing cancer risk in T2DM, particularly individual cancer types where the number of cases is low and statistical conclusion is not possible. Pre-clinical studies indicated that dapagliflozin is not carcinogenic. In addition to rigorously demonstrating that dapagliflozin is not an initiator of tumor development, neither the drug, its primary metabolite, nor drug-induced glucosuria have been found to enhance the growth of human bladder cancer cells in vitro or in a mouse xenograft model [30, 33]. Further, exposure to dapagliflozin in the presence of extensive glucosuria did not lead to the conversion of hyperplastic cells to bladder tumors. Therefore, the possibility of tumor cells being more susceptible to the potential growth effects of glucosuria seems unlikely.

These findings are in contrast with studies of known carcinogenic agents such as estrogen, for which several mechanisms are postulated and where there is a clear link to breast cancer in humans [42]. Pre-clinical data have shown an association between pioglitazone exposure and bladder tumors in male rats; tumor formation was considered secondary to the formation of urinary solids with subsequent irritation and hyperplasia [43]. A similar rat-specific mode of action for urinary bladder tumors was demonstrated by the PPAR $\gamma / \alpha$ agonist, muraglitazar [44, 45]. Further, as described earlier, other less selective SGLT2 inhibitors have been shown to induce tumors (although not urothelial tumors of the kidney pelvis, ureters, or bladder) in animal studies [27, 28], but the associated mechanisms are considered irrelevant to humans. Such findings therefore bring SGLT2 inhibitors under increased scrutiny for their carcinogenic potential.

This post hoc pooled analysis of dapagliflozin clinical trials showed no obvious pattern in the distribution of various tumors, with no statistically significant differences between treatment groups for any tumor types. The number of bladder cancer cases was small, but numerical imbalances were noted between treatment groups [46]. It should be noted that the characteristics of patients reporting urothelial cell carcinomas during the study period were $\geq 60$ years old, male, and most were current or former smokers and typical of the general population. Although it does not explain the greater number of cancer cases in patients treated with dapagliflozin versus comparator, these data highlight that some well-established and potent risk factors of bladder cancer in patients with T2DM were present, which cannot be ignored.

The assessment of incidence rates in a clinical trial versus real-world population has its own challenges. The dapagliflozin trials were not large enough and were not designed to assess the risk of bladder cancer (as noted by the wide $95 \%$ CIs), and it is questionable whether data reported in the dapagliflozin trials can be extrapolated to a larger, "real life" population of patients with bladder cancer. Furthermore, the incidence rates for bladder cancer in the general population vary among different countries and between databases-this could potentially be related to an absence of routine screening for bladder cancer detection. 
The assessment of bladder cancer with dapagliflozin may also potentially be confounded because of the drug-induced diuresis and symptomatology associated with genitourinary infections. This could have possibly prompted more rigorous investigations in the dapagliflozin treatment groups, leading to detection of pre-existing bladder cancers. However, this hypothesis cannot be confirmed because of the absence of systematic collection of data for medical procedures related to cancer diagnosis in these trials.

Previous studies have shown an association between recurrent UTIs and bladder cancer [47, 48], and therefore one important question was whether the bladder cancer cases with dapagliflozin were related to increased rates of UTIs. However, of the ten patients reporting bladder cancer in the dapagliflozin clinical development program, seven were not preceded by urinary events. Furthermore, other SGLT2 inhibitors, which are associated with very similar patterns of infection, were not associated with bladder cancer imbalances in clinical trials. Hence, these observations do not support a causal relationship between UTIs in dapagliflozin-treated patients and bladder cancer.

Bladder carcinomas usually have a latency period of several years [34]. In one study, cyclophosphamide, a well-described bladder carcinogen, was administered to patients with Wegener's syndrome with a median drug exposure of 2.7 years. The estimated incidence of bladder cancer after the first exposure to cyclophosphamide was $5 \%$ at 10 years [49]. In contrast, the time from initiation of dapagliflozin to bladder cancer development was much shorter, making it unlikely that the exposure to dapagliflozin in studies to date would have been long enough to produce a quantifiable effect on bladder tumor development or growth.

Unlike in daily practice, urinalysis is often conducted in clinical trials, making the detection of hematuria more likely. However, there was an absence of detailed workup of hematuria prior to randomization in the dapagliflozin trials, which is typical of clinical practice [50]. No specific direction was provided and no hematuria workup data were collected proactively until later studies, where local guidance was followed. The management and follow-up of hematuria are often ambiguous due to lack of clarity surrounding definitions, diagnosis, and referral criteria. Studies have suggested that most patients with hematuria are not appropriately referred to urologists for evaluation [51-53]. Failure to exclude bladder cancer prior to randomization increases the likelihood of recruiting patients with pre-existing bladder cancer in clinical trials or may delay the final diagnosis to after the study initiation. In the present analysis, all except one patient had hematuria prior to the start of treatment or within 6 months of initiating the study drug, and it took several additional weeks or months to establish the final diagnosis of bladder cancer. Such findings highlight an unmet need for improved guidance regarding a complete workup of hematuria or any other potential indicators of pre-existing cancers for clinical trials conducted in patients with T2DM. It is important to note that in clinical practice, the use of dapagliflozin should not be precluded in patients presenting with hematuria, and neither is it necessary to routinely screen for hematuria before or during dapagliflozin treatment.

In summary, the totality of evidence to date does not suggest a causal relationship between dapagliflozin and bladder cancer, and the observed effect in clinical trials may be a 
chance finding. However, given the observed imbalances, any firm conclusion cannot be made without additional clinical data, and as such accruing bladder cancer events are being carefully investigated in ongoing trials of dapagliflozin.

\section{CONCLUSION}

The case of dapagliflozin illustrates the challenges of interpreting bladder cancer events with a drug associated with urinary symptoms and in a disease where the background incidence of bladder cancer is elevated. An important learning from the dapagliflozin experience is the need for improved and comprehensive follow-up of hematuria in clinical trials at baseline and throughout the study period, and in real-world populations.

\section{ACKNOWLEDGMENTS}

All authors meet the ICMJE criteria for authorship for this manuscript, take responsibility for the integrity of the work as a whole, and have given final approval to the version to be published. This study and the article processing charges were funded by AstraZeneca. Medical writing support was provided by Shelley Narula and Mark Davies of inScience Communications, Springer Healthcare Ltd, funded by AstraZeneca.

Conflict of interest. SMC is a consultant for AstraZeneca and Bristol-Myers Squibb (dapagliflozin), Johnson \& Johnson (canagliflozin) and Boehringer Ingelheim (empagliflozin). EMM declares that he has no conflict of interest. AP and TPR are employees of
Bristol-Myers Squibb. EJ and KJ are employees of AstraZeneca.

Compliance with ethic guidelines. The analysis in this article is based on previously conducted studies and does not involve any new studies of human or animal subjects performed by any of the authors.

Open Access. This article is distributed under the terms of the Creative Commons Attribution-NonCommercial 4.0 International License (http://creativecommons.org/licenses/ by-nc/4.0/), which permits any noncommercial use, distribution, and reproduction in any medium, provided you give appropriate credit to the original author(s) and the source, provide a link to the Creative Commons license, and indicate if changes were made.

\section{REFERENCES}

1. Giovannucci E, Harlan DM, Archer MC, et al. Diabetes and cancer: a consensus report. Diabetes Care. 2010;33:1674-85.

2. Fang H, Yao B, Yan Y, et al. Diabetes mellitus increases the risk of bladder cancer: an updated meta-analysis of observational studies. Diabetes Technol Ther. 2013;15:914-22.

3. Zhu Z, Wang X, Shen Z, Lu Y, Zhong S, Xu C. Risk of bladder cancer in patients with diabetes mellitus: an updated meta-analysis of 36 observational studies. BMC Cancer. 2013;13:310.

4. Newton CC, Gapstur SM, Campbell PT, Jacobs EJ. Type 2 diabetes mellitus, insulin-use and risk of bladder cancer in a large cohort study. Int J Cancer. 2013;132:2186-91.

5. MacKenzie T, Zens MS, Ferrara A, Schned A, Karagas MR. Diabetes and risk of bladder cancer: evidence from a case-control study in New England. Cancer. 2011;117:1552-6.

6. Suissa S, Azoulay L, Dell'Aniello S, Evans M, Vora J, Pollak M. Long-term effects of insulin glargine on the risk of breast cancer. Diabetologia. 2011;54:2254-62. 
7. Hanefeld M, Bramlage P. Insulin use early in the course of type 2 diabetes mellitus: the ORIGIN trial. Curr Diab Rep. 2013;13:342-9.

8. Hemkens LG, Grouven U, Bender R, et al. Risk of malignancies in patients with diabetes treated with human insulin or insulin analogues: a cohort study. Diabetologia. 2009;52:1732-44.

9. Ruiter R, Visser LE, van Herk-Sukel MP, et al. Risk of cancer in patients on insulin glargine and other insulin analogues in comparison with those on human insulin: results from a large population-based follow-up study. Diabetologia. 2012;55:51-62.

10. Macconell L, Brown C, Gurney K, Han J. Safety and tolerability of exenatide twice daily in patients with type 2 diabetes: integrated analysis of 5594 patients from 19 placebo-controlled and comparator-controlled clinical trials. Diabetes Metab Syndr Obes. 2012;5:29-41.

11. Monami M, Dicembrini I, Nardini C, Fiordelli I, Mannucci E. Glucagon-like peptide-1 receptor agonists and pancreatitis: a meta-analysis of randomized clinical trials. Diabetes Res Clin Pract. 2014;103:269-75.

12. Li L, Shen J, Bala MM, et al. Incretin treatment and risk of pancreatitis in patients with type 2 diabetes mellitus: systematic review and meta-analysis of randomised and non-randomised studies. BMJ. 2014;348:g2366.

13. Roy D, Chadwick KD, Tatarkiewicz K, et al. The glucagon-like peptide-1-based therapeutics exenatide and saxagliptin did not cause detrimental effects on the pancreas in mice, rats, dogs and monkeys. Diabetes Obes Metab. 2014;16:910-21.

14. Alves C, Batel-Marques F, Macedo AF. A meta-analysis of serious adverse events reported with exenatide and liraglutide: acute pancreatitis and cancer. Diabetes Res Clin Pract. 2012;98:271-84.

15. FDA Drug Safety Communication. Ongoing safety review of actos (pioglitazone) and potential increased risk of bladder cancer after two years exposure [database on the Internet] 2010. Available from: http://www.fda.gov/drugs/drugsafety/ucm22 6214.htm. Accessed May 7, 2014.

16. US Food and Drug Administration. FDA Drug Safety Podcast for Healthcare Professionals: update to ongoing safety review of Actos (pioglitazone) and increased risk of bladder cancer. 2013. Available from: http://www.fda.gov/Drugs/DrugSafety/Drug SafetyPodcasts/ucm259489.htm. Accessed Aug 2015.
17. Lewis JD, Ferrara A, Peng T, et al. Risk of bladder cancer among diabetic patients treated with pioglitazone: interim report of a longitudinal cohort study. Diabetes Care. 2011;34:916-22.

18. Takeda Pharmaceuticals International GmbH. Takeda Announces Completion of the Post-Marketing Commitment to Submit Data to the FDA, the EMA and the PMDA for Pioglitazone Containing Medicines Including ACTOS. 2014. Available from: https://www.takeda.com/news/ 2014/20140829_6714.html. Accessed Aug 10, 2015.

19. Wei L, MacDonald TM, Mackenzie IS. Pioglitazone and bladder cancer: a propensity score matched cohort study. Br J Clin Pharmacol. 2013;75:254-9.

20. Balaji V, Seshiah V, Ashtalakshmi G, Ramanan SG, Janarthinakani $M$. A retrospective study on finding correlation of pioglitazone and incidences of bladder cancer in the Indian population. Indian J Endocrinol Metab. 2014;18:425-7.

21. Abdul-Ghani MA, DeFronzo RA. Inhibition of renal glucose reabsorption: a novel strategy for achieving glucose control in type 2 diabetes mellitus. Endocr Pract. 2008;14:782-90.

22. Farxiga (dapagliflozin). Highlights of prescribing information. Farxiga (dapagliflozin) tablets, for oral use. Princeton, NJ. 2014. Available from: http:// www.accessdata.fda.gov/drugsatfda_docs/label/ 2014/202293s003lbl.pdf. Accessed Aug 10, 2015.

23. European Medicines Agency. EPAR summary for the public. [database on the Internet] 2012. Available from: http://www.ema.europa.eu/docs/en_GB/doc ument_library/EPAR_-_Summary_for_the_public/ human/002322/WC500136025.pdf. Accessed May $15,2014$.

24. Prescribing information [database on the Internet] 2014. Available from: http://www.azpicentral.com/ xigduo/pi_xigduoxr.pdf. Accessed Jun 2015.

25. Xigduo: EPAR. Product information [database on the Internet] 2014. Available from: http://www. ema.europa.eu/docs/en_GB/document_library/ EPAR_-_Product_Information/human/002672/ WC500161033.pdf. Accessed Jun 2015.

26. Dapagliflozin. Background document [database on the Internet] 2011. Available from: http://www.fda. gov/downloads/AdvisoryCommittees/Committees MeetingMaterials/Drugs/EndocrinologicandMeta bolicDrugsAdvisoryCommittee/UCM262996.pdf. Accessed Jun 2015.

27. De Jonghe $S$, Proctor J, Vinken $P$, et al. Carcinogenicity in rats of the SGLT2 inhibitor canagliflozin. Chem Biol Interact. 2014;224:1-12. 
28. Taub ME, Ludwig-Schwellinger E, Ishiguro N, et al. Sex-, species-, and tissue-specific metabolism of empagliflozin in male mouse kidney forms an unstable hemiacetal metabolite (M466/2) that degrades to 4-Hydroxycrotonaldehyde, a reactive and cytotoxic species. Chem Res Toxicol. 2015;28:103-15.

29. Abdul-Ghani MA, Norton L, DeFronzo RA. Efficacy and safety of SGLT2 inhibitors in the treatment of type 2 diabetes mellitus. Curr Diab Rep. 2012;12:230-8.

30. Tirmenstein M, Dorr TE, Janovitz EB, et al. Nonclinical toxicology assessments support the chronic safety of dapagliflozin, a first-in-class sodium-glucose cotransporter 2 inhibitor. Int $\mathrm{J}$ Toxicol. 2013;32:336-50.

31. List JF, Woo V, Morales E, Tang W, Fiedorek FT. Sodium-glucose cotransport inhibition with dapagliflozin in type 2 diabetes. Diabetes Care. 2009;32:650-7.

32. Center for Drug Evaluation and Research. Pharmacology/Toxicology NDA review and evaluation. 2013. Available from: http://www. accessdata.fda.gov/drugsatfda_docs/nda/2014/2022 93Orig1s000PharmR.pdf. Accessed Aug 10, 2015.

33. Reilly TP, Graziano MJ, Janovitz EB, et al. Carcinogenicity risk assessment supports the chronic safety of dapagliflozin, an inhibitor of sodium-glucose co-transporter 2 , in the treatment of type 2 diabetes mellitus. Diabetes Ther. 2014;5:73-96.

34. Clayson DB, Cooper EH. Cancer of the urinary tract. Adv Cancer Res. 1970;13:271-381.

35. Tian L, Cai T, Pfeffer MA, Piankov N, Cremieux PY, Wei LJ. Exact and efficient inference procedure for meta-analysis and its application to the analysis of independent $2 \times 2$ tables with all available data but without artificial continuity correction. Biostatistics. 2009;10:275-81.

36. Kobayashi H, Kikuchi E, Mikami S, et al. Long term follow-up in patients with initially diagnosed low grade Ta non-muscle invasive bladder tumors: tumor recurrence and worsening progression. BMC Urol. 2014;14:5.

37. Crow P, Bayley J, Keeley F Jr. Smoking and urological disease. BJU Int. 2009;103:1317-9.

38. Baris D, Karagas MR, Verrill C, et al. A case-control study of smoking and bladder cancer risk: emergent patterns over time. J Natl Cancer Inst. 2009;101:1553-61.

39. Matanoski GM, Elliott EA. Bladder cancer epidemiology. Epidemiol Rev. 1981;3:203-29.
40. Travis LB, Curtis RE, Glimelius B, et al. Bladder and kidney cancer following cyclophosphamide therapy for non-Hodgkin's lymphoma. J Natl Cancer Inst. 1995;87:524-30.

41. Friedman GD, Carroll PR, Cattolica EV, Hiatt RA. Can hematuria be a predictor as well as a symptom or sign of bladder cancer? Cancer Epidemiol Biomarkers Prev. 1996;5:993-6.

42. Malone KE. Diethylstilbestrol (DES) and breast cancer. Epidemiol Rev. 1993;15:108-9.

43. European Medicines Agency. Actos: EPAR. 2003. Available from: http://www.ema.europa.eu/docs/ en_GB/document_library/EPAR_-_Product_Informa tion/human/000285/WC500021386.pdf. Accessed Aug 10, 2015.

44. Waites CR, Dominick MA, Sanderson TP, Schilling BE. Nonclinical safety evaluation of muraglitazar, a novel PPARalpha/gamma agonist. Toxicol Sci. 2007;100:248-58.

45. Tseng $\mathrm{CH}$, Tseng FH. Peroxisome proliferator-activated receptor agonists and bladder cancer: lessons from animal studies. J Environ Sci Health C Environ Carcinog Ecotoxicol Rev. 2012;30:368-402.

46. SEER Stat Fact Sheets: Bladder Cancer [database on the Internet] 2011. Available from: http://seer. cancer.gov/statfacts/html/urinb.html. Accessed Mar 24, 2015.

47. Shephard EA, Stapley S, Neal RD, Rose P, Walter FM, Hamilton WT. Clinical features of bladder cancer in primary care. $\mathrm{Br} J$ Gen Pract. 2012;62:e598-604.

48. Vermeulen SH, Hanum N, Grotenhuis AJ, et al. Recurrent urinary tract infection and risk of bladder cancer in the Nijmegen bladder cancer study. Br J Cancer. 2015;112:594-600.

49. Talar-Williams C, Hijazi YM, Walther MM, et al. Cyclophosphamide-induced cystitis and bladder cancer in patients with Wegener granulomatosis. Ann Intern Med. 1996;124:477-84.

50. Friedlander DF, Resnick MJ, You C, et al. Variation in the intensity of hematuria evaluation: a target for primary care quality improvement. Am J Med. 2014;127:633-40.

51. Johnson EK, Daignault S, Zhang Y, Lee CT. Patterns of hematuria referral to urologists: does a gender disparity exist? Urology. 2008;72:498-502 (discussion 502-3).

52. Nieder AM, Lotan Y, Nuss GR, et al. Are patients with hematuria appropriately referred to Urology? 
A multi-institutional questionnaire based survey. Urol Oncol. 2010;28:500-3.

53. Shinagare AB, Silverman SG, Gershanik EF, Chang SL, Khorasani R. Evaluating hematuria: impact of guideline adherence on urologic cancer diagnosis. Am J Med. 2014;127:625-32.

54. Kaku K, Inoue S, Matsuoka O, et al. Efficacy and safety of dapagliflozin as a monotherapy for type 2 diabetes mellitus in Japanese patients with inadequate glycaemic control: a phase II multicentre, randomized, double-blind, placebo-controlled trial. Diabetes Obes Metab. 2013;15:432-40.

55. Rosenstock J, Vico M, Wei L, Salsali A, List JF. Effects of dapagliflozin, an SGLT2 inhibitor, on $\operatorname{HbA}(1 \mathrm{c})$, body weight, and hypoglycemia risk in patients with type 2 diabetes inadequately controlled on pioglitazone monotherapy. Diabetes Care. 2012;35:1473-8.

56. Wilding JP, Woo V, Soler NG, et al. Long-term efficacy of dapagliflozin in patients with type 2 diabetes mellitus receiving high doses of insulin: a randomized trial. Ann Intern Med. 2012;156:405-15.

57. Bailey CJ, Gross JL, Hennicken D, Iqbal N, Mansfield TA, List JF. Dapagliflozin add-on to metformin in type 2 diabetes inadequately controlled with metformin: a randomized, double-blind, placebo-controlled 102-week trial. BMC Med. 2013;11:43.

58. Nauck MA, Del Prato S, Meier JJ, et al. Dapagliflozin versus glipizide as add-on therapy in patients with type 2 diabetes who have inadequate glycemic control with metformin: a randomized, 52-week, double-blind, active-controlled noninferiority trial. Diabetes Care. 2011;34:2015-22.

59. Leiter LA, Cefalu WT, de Bruin TW, Gause-Nilsson I, Sugg J, Parikh SJ. Dapagliflozin added to usual care in individuals with type 2 diabetes mellitus with preexisting cardiovascular disease: a 24-week, multicenter, randomized, double-blind, placebo-controlled study with a 28-week extension. J Am Geriatr Soc. 2014;62:1252-62. 